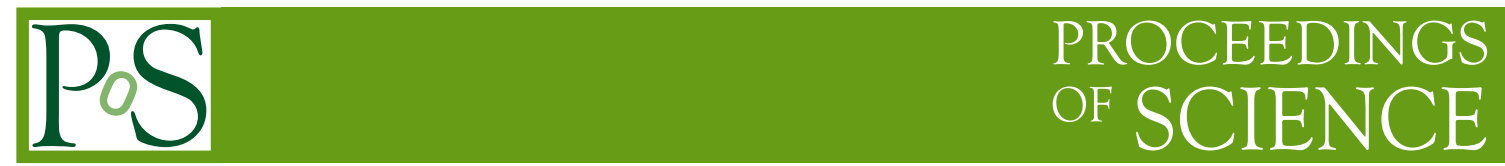

\title{
Strangeness production at HERA
}

\author{
Armen Buniatyan* \\ Physics Institute of University of Heidelberg \\ E-mail: Armen. Buniatyanedesy.de \\ On behalf of the HI and ZEUS Collaborations
}

The recent results on strange hadron production from H1 and ZEUS Experiments at HERA are discussed. The Monte Carlo and theoretical models are compared to the measurements.

International Conference on the Structure and the Interactions of the Photon including the 20th International Workshop on Photon-Photon Collisions and the International Workshop on High Energy Photon Linear Colliders

20 - 24 May 2013

Paris, France

${ }^{*}$ Speaker. 


\section{Introduction}

The measurements of strange particle production in high energy collisions provide valuable information for understanding Quantum Chromodynamics (QCD) in the perturbative and nonperturbative regimes. In neutral current deep-inelastic ep scattering (DIS) four different processes depicted in Figure 1 contribute to strange hadron production. Strange quarks may originate di-

(a)

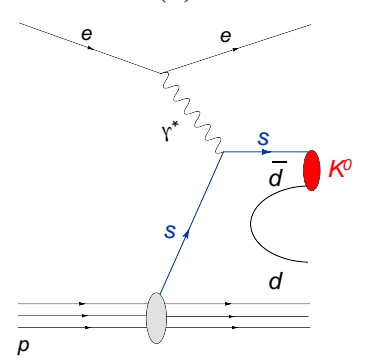

(b)

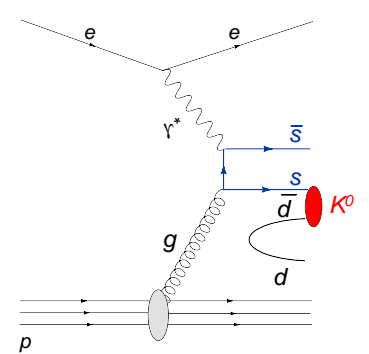

(c)

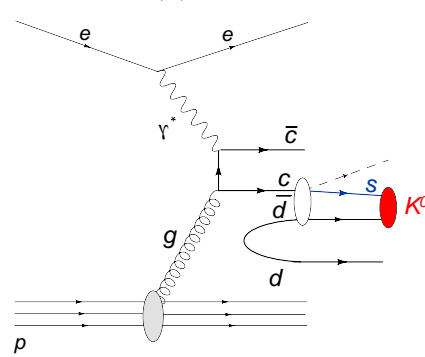

(d)

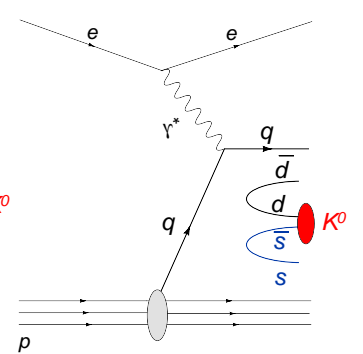

Figure 1: Schematic diagrams for the processes contributing to strangeness production in $e p$ scattering: (a) direct production from the strange sea, (b) BGF, (c) heavy hadron decays and (d) fragmentation.

rectly from the strange sea of the proton (Figure 1a), from boson-gluon-fusion (BGF, Figure 1b), from the decays of heavy flavoured hadrons (Figure 1c) and from the creation of $s \bar{s}$ pairs in the non-perturbative fragmentation process (Figure 1d). The latter process is the dominant source for strange hadron production.

The jet fragmentation and hadronisation processes through which coloured partons become bound in colour-neutral hadrons cannot be described within the framework of perturbative QCD (pQCD). Several approaches have been developed which attempt to build a bridge between the fixed-order partonic cross sections and the observed hadrons. Two of the most successful and widely used approaches are the Lund string model [1] and the fragmentation functions (FFs) [2-6]. The Lund string model, relying on a large number of parameters, is interfaced to leading-logarithm parton-shower Monte Carlo (MC) models. The FFs are parameterisations of the hadronisation process within the standard framework of leading-twist collinear QCD factorisation, in a similar way to that of the parton distribution functions (PDFs), and are convoluted with the predicted partonic cross sections.

In this report the new mesurements of $K_{s}^{0}$ and $\Lambda$ production performed by the H1 and ZEUS experiments at the electron-proton storage ring HERA are presented [7, 8, 9].

\section{2. $K_{s}^{0}$ and $\Lambda$ production cross sections}

The kinematics of the electron-proton scattering process is described using three Lorentz invariant variables: the photon virtuality, $Q^{2}$, the inelasticity in the proton rest frame $y$ and the Bjorken scaling variable $x$. Because of the relation $Q^{2}=x y s$ at fixed $\sqrt{s}$ only two of these variables are independent.

The H1 Collaboration presented new measurements of $K_{s}^{0}$ production at $7<Q^{2}<100 \mathrm{GeV}^{2}$ [7] and $\Lambda$ production at $145<Q^{2}<20000 \mathrm{GeV}^{2}$ [8]. The data used in the analyses correspond to an integrated luminosity of $109 \mathrm{pb}^{-1}$ in case of $K_{s}^{0}$ production and $340 \mathrm{pb}^{-1}$ in case of $\Lambda$ production 

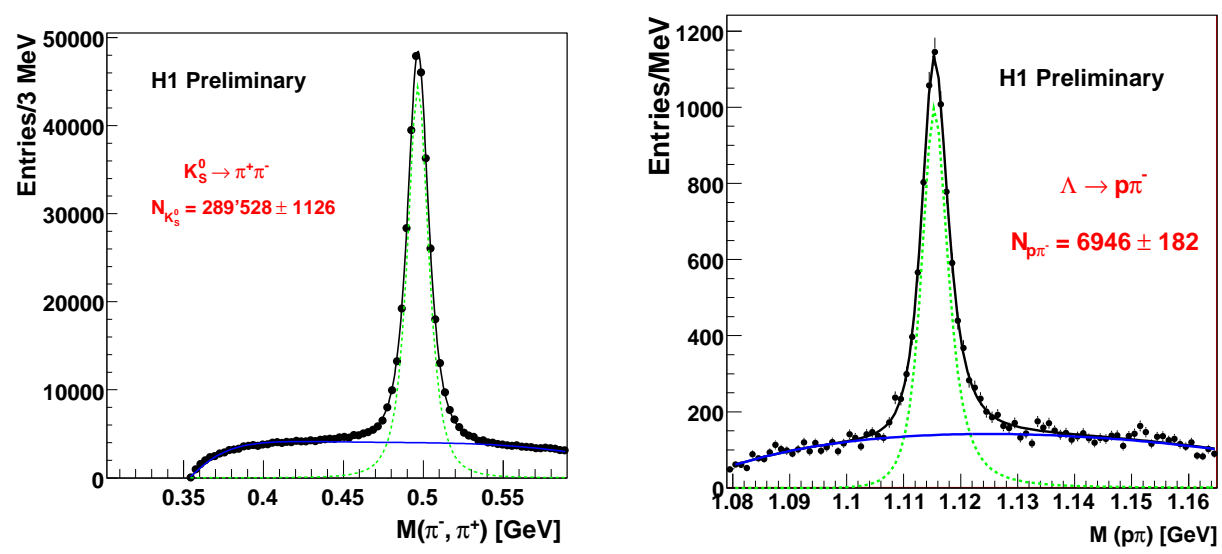

Figure 2: Invariant mass distribution of $K_{s}^{0}$ candidates from $\pi^{+} \pi^{-}$and $\Lambda$ candidates from $p \pi^{-}$.

and were collected with the H1 detector in the years 2004 to 2007 when protons with an energy of $920 \mathrm{GeV}$ collided with electrons with an energy of $27.6 \mathrm{GeV}$ producing a centre-of-mass energy of $\sqrt{s}=319 \mathrm{GeV}$.

The $K_{s}^{0}$ mesons and $\Lambda$ baryons are measured by the kinematic reconstruction of their decays $K_{s}^{0} \rightarrow \pi^{+} \pi^{-}$and $\Lambda \rightarrow p \pi^{-}$, respectively. The number of $K_{s}^{0}$ mesons and $\Lambda$ baryons is obtained by fitting the invariant mass spectra with the sum of a signal and background function. For the signal function the skewed t-student function is used while the background shape is described by a threshold function with exponential damping. In total approximately 290,000 $K_{s}^{0}$ mesons and 7,000 $\Lambda$ or $\bar{\Lambda}$ baryons are reconstructed in the phase space given in Table 1 . The fitted $K_{s}^{0}$ and $\Lambda$ masses agree with the world average [10].

\begin{tabular}{|c|c|c|}
\hline & DIS kinematics & Hadron kinematics \\
\hline$K_{s}^{0}$ & $7<Q^{2}<100 \mathrm{GeV}^{2}, 0.1<y<0.6$ & $0.5<p_{T}<3.5 \mathrm{GeV},-1.3<\eta<1.3$ \\
\hline$\Lambda$ & $145<Q^{2}<20000 \mathrm{GeV}^{2}, 0.2<y<0.6$ & $p_{T}>0.3 \mathrm{GeV},-1.5<\eta<1.5$ \\
\hline
\end{tabular}

Table 1: Phase space regions explored in the analyses of $K_{s}^{0}$ and $\Lambda$ production, respectively.

The visible inclusive production cross sections $\sigma_{v i s}$ measured in the kinematic region defined in Table 1, are

$$
\begin{aligned}
\sigma_{v i s}\left(e p \rightarrow e K_{s}^{0} X\right) & =10.66 \pm 0.02(\text { stat. })_{-8.5}^{+9.4} \text { (syst.) nb } \\
\sigma_{v i s}(e p \rightarrow e \Lambda X) & =144.7 \pm 0.04(\text { stat. })_{-8.5}^{+9.4} \text { (syst.) pb. }
\end{aligned}
$$

The Monte Carlo model predictions are compared to the measurements. The DIS process is modelled using the RAPGAP [11] and the DJANGOH [12] programs, which generate hard partonic processes at the Born level at leading order in $\alpha_{s}$, convoluted with the PDF of the proton. Two different approaches are used for the simulation of higher order QCD effects: in RAPGAP the parton shower approach (MEPS) is implemented in which the parton emission is ordered in transverse 


\begin{tabular}{|c|c|c|}
\hline & $\lambda_{s}=0.220$ & $\lambda_{s}=0.286$ \\
\hline$\sigma_{v i s}(e p \rightarrow e[\Lambda+\bar{\Lambda}] X) \mathrm{CDM}$ & $136 \mathrm{pb}$ & $161 \mathrm{pb}$ \\
\hline$\sigma_{v i s}(e p \rightarrow e[\Lambda+\bar{\Lambda}] X) \mathrm{MEPS}$ & $120 \mathrm{pb}$ & $144 \mathrm{pb}$ \\
\hline
\end{tabular}

Table 2: Monte Carlo predictions for different settings of the strangeness suppresion factor $\lambda_{s}$.

momentum $k_{T}$ according to the leading-log approximation; and in DJANGOH the colour dipol approach (CDM) [13] as implemented in ARIADNE [14] is adopted in which partons are created by colour dipole radiation between the partons in the cascade, resulting in a $k_{T}$ unordered parton emission.

In the modelling of the fragmentation process the suppression of $s \bar{s}$ pairs due to the mass of the strange quark is generally controlled by the strangeness suppression factor $\lambda_{s}[1,15]$. Especially, the ratio of $K_{s}^{0}$ to charged particles should strongly depends on this quark mass effect. Using a strangeness suppression factor of $\lambda_{s}=0.286$ the models RAPGAP (MEPS) and DJANGOH $(\mathrm{CDM})$ predict $K_{s}^{0}$ cross sections of $10.93 \mathrm{nb}$ and $9.88 \mathrm{nb}$, respectively, in reasonable agreement with the measurement. The cross section predictions for $\Lambda+\bar{\Lambda}$ production from the MEPS and CDM [13] models are shown in Table 2 for two values of the strangeness suppression parameter $\lambda_{s}$. The measured inclusive $\Lambda+\bar{\Lambda}$ cross section is close to the CDM prediction with $\lambda_{s}=0.22$ and to the MEPS prediction with $\lambda_{s}=0.286$.
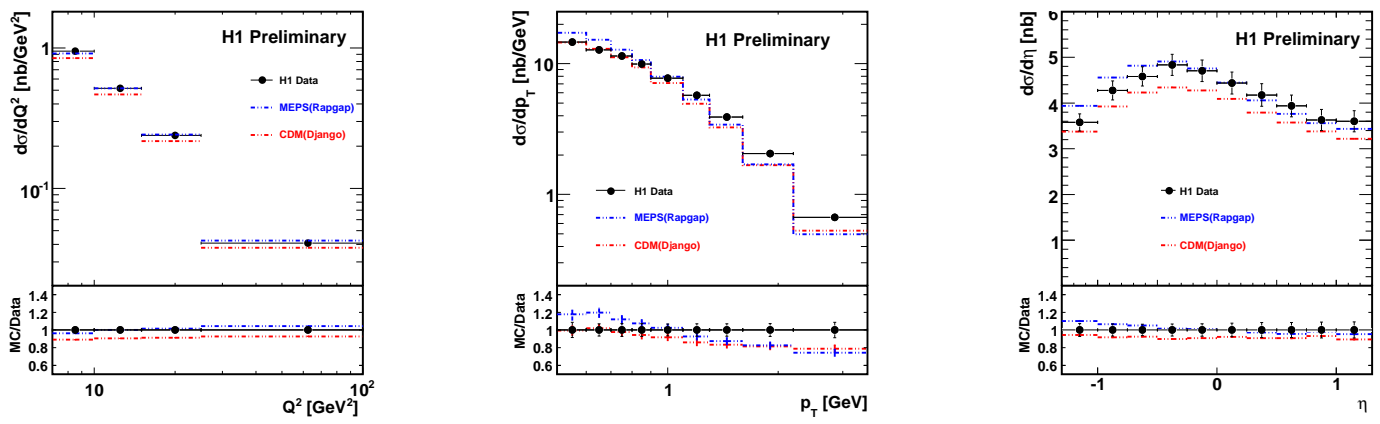

Figure 3: Differential $K_{s}^{0}$ production cross sections as a function of the photon virtuality squared $Q^{2}$, transverse momentum $p_{T}$ and its pseudorapidity $\eta$ in comparison to RAPGAP (MEPS) and DJANGOH (CDM).

Differential cross sections of $K_{s}^{0}$ and $\Lambda$ production are shown in Figures 3 and 4 as a function of $Q^{2}$, the transverse momentum $p_{T}$ and the pseudorapidity $\eta$ measured in the laboratory frame along with the predictions of the MEPS and CDM models. The cross sections fall rapidly as $Q^{2}$ grows. Apart from small normalisation differences the models describe the shapes of the measured cross sections as a function of $Q^{2}$ and $\eta$ reasonably well.

\subsection{Ratio of $K_{s}^{0}$ production to charged particle production}

By normalising the $K_{s}^{0}$ production cross section to the cross section of charged particle production many model dependent uncertainties, like the cross section dependence on proton PDFs, 

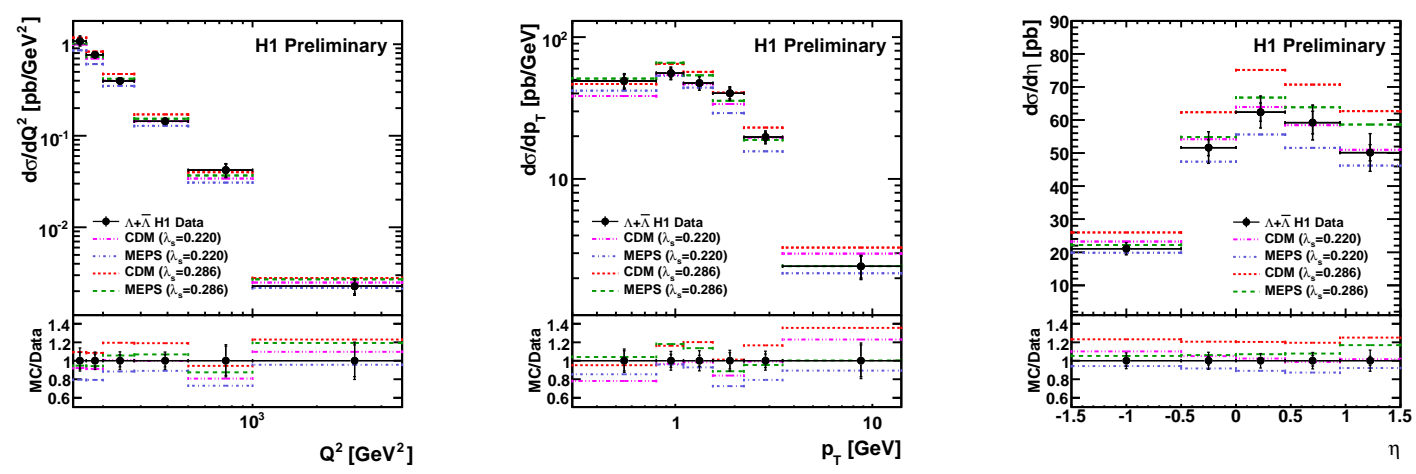

Figure 4: Differential $\Lambda$ production cross sections as a function of the photon virtuality squared $Q^{2}$, transverse momentum $p_{T}$ and its pseudorapidity $\eta$ in comparison to RAPGAP (MEPS) and DJANGOH (CDM).

cancel thus enhancing the sensitivity to details of the fragmentation process. In Figure 5 the ratio of $K_{s}^{0}$ production to the cross section charged particle procduction is shown as a function of $p_{T}$ and $\eta$ and in comparison to the expectations from DJANGOH using three different values of $\lambda_{s}$ ranging from 0.220 to 0.35 . The ratios are well described by the model in shape and a high sensitivity on $\lambda_{s}$ is observed in the absolute value of this ratio, demonstrating the clear potential of using this ratio for extracting the strangeness suppression factor $\lambda_{s}$.
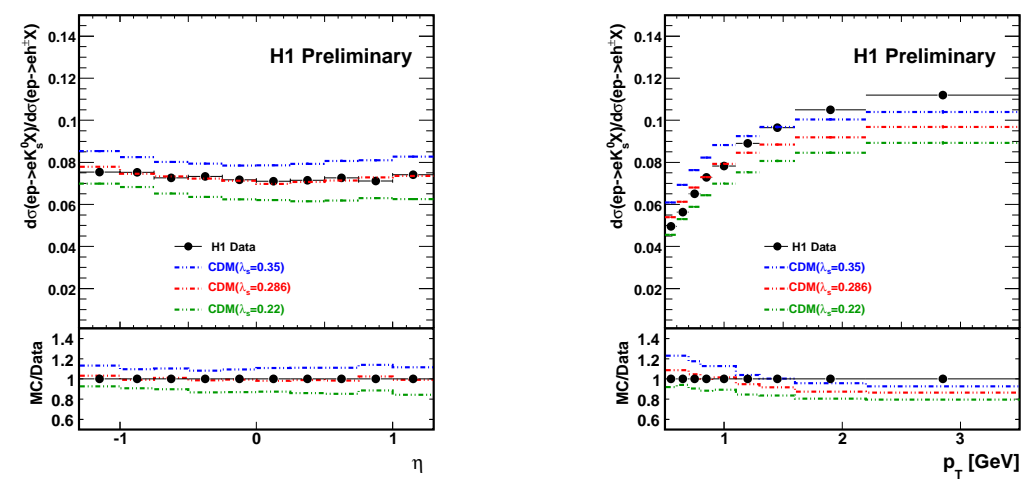

Figure 5: Ratio of $K_{s}^{0}$ to charged particle production cross sections as a function of pseudorapidity $\eta$ and transverse momentum $p_{T}$ in comparison to DJANGOH (CDM) for three different vaues of $\lambda_{s}$.

\section{2 $\Lambda$ Production to DIS Cross Section Ratio}

In the left side of Figure 6 the ratio of $\Lambda$ production to DIS cross section is shown as a function of $Q^{2}$ in comparison to the expectations from RAPGAP and DJANGOH both using $\lambda_{s}=0.286$ and $\lambda_{s}=0.220$. The DJANGOH prediction with $\lambda_{s}=0.286$ yields the worst description of the data by overshooting them significantly. For the same strangeness suppression factor also RAPGAP tends to yield ratios larger than observed in data for $Q^{2}<200 \mathrm{GeV}^{2}$. The best description is provided by DJANGHO using $\lambda_{s}=0.220$. 

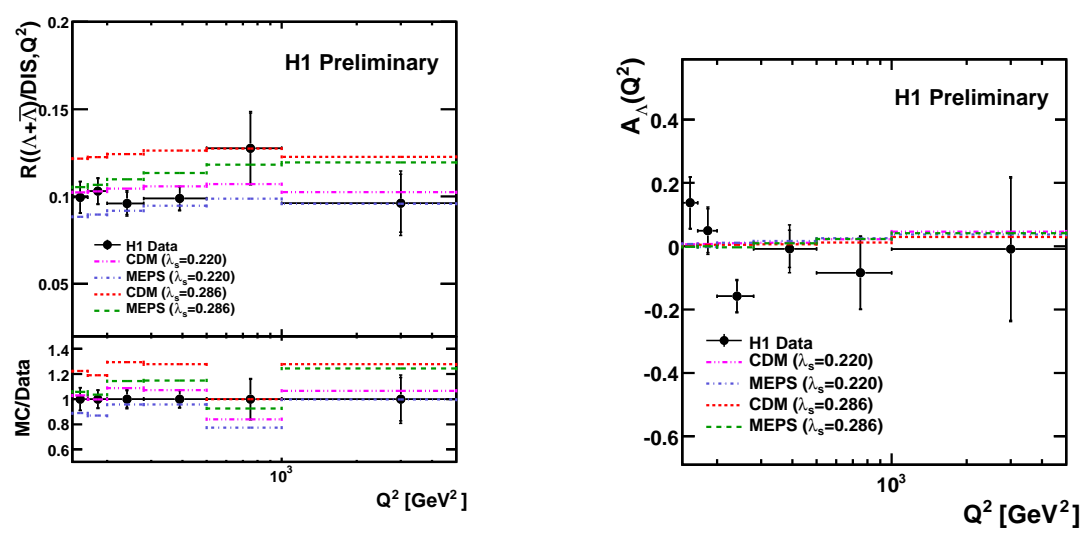

Figure 6: (Left) Ratio $R(D I S)$ of $\Lambda$ production cross section to DIS cross section as a function of $Q^{2}$ and Bjorken $x$ variable in comparison to RAPGAP (MEPS) and DJANGOH (CDM) with two different vaues of $\lambda_{s}$. (Right) Asymmetry $A_{\Lambda}$ as a function of $Q^{2}$ in comparison to RAPGAP (MEPS) and DJANGOH (CDM) with two different vaues of $\lambda_{s}$.

\section{$2.3 \Lambda-\bar{\Lambda}$ Asymmetries}

The asymmetry $\Lambda-\bar{\Lambda}$ which is defined as

$$
A_{\Lambda}=\frac{\sigma_{v i s}(e p \rightarrow e \Lambda X)-\sigma_{v i s}(e p \rightarrow e \bar{\Lambda} X)}{\sigma_{v i s}(e p \rightarrow e \Lambda X)+\sigma_{v i s}(e p \rightarrow e \bar{\Lambda} X)} .
$$

may shed light on the mechanism of baryon number transfer in $e p$ scattering. A significant positive asymmetry would be an indication for the baryon number transfer from the proton to the $\Lambda$ baryon. If present such an effect should be more pronounced in the positive $\eta$ region in the laboratory frame. For the kinemaic region defined in Table 1 the asymmetry is measured to be

$$
A_{\Lambda}=0.002 \pm 0.022 \text { (stat.) } \pm 0.018 \text { (syst.). }
$$

In the right side of Figure 6 the asymmetry $A_{\Lambda}$ is shown as a function of $Q^{2}$. The data show no evidence for a non-vanishing asymmetry in the phase space region investigated.

\section{Scaled momentum distribution of $K_{s}^{0}$ and $\Lambda$ in DIS}

Extensive studies of the fragmentation properties of the hadronic final state have been performed in $e^{+} e^{-}, p p, p \bar{p}$ and deep inelastic ep scattering data and have provided information about the fragmentation and hadronisation processes. The comparison of the measurements in different reactions indicated an approximately universal behaviour of quark fragmentation.

The parameterisations $[16,17,18]$ of the FFs for strange hadrons are so far largely unconstrained. The HERA data have the potential to constrain these FFs over a wide kinematic range. Here a new measurement of ZEUS Collaboration of the scaled momentum distributions for $K_{S}^{0}$ and $\Lambda$ hadrons in DIS [9] is presented. The data used in this analysis were collected during the running period 2005-2007, when HERA operated with protons of energy $E_{p}=920 \mathrm{GeV}$ and electrons of energy $E_{e}=27.5 \mathrm{GeV}$, and correspond to an integrated luminosity of $330 \mathrm{pb}^{-1}$. 
The scaled momentum is defined as $x_{p}=2 P^{\text {Breit }} / \sqrt{Q^{2}}$, where $P^{\text {Breit }}$ is the particle momentum in the Breit frame $[19,20]$. The measurements are performed in the current region of the Breit frame, which is equivalent to one hemisphere in $e^{+} e^{-}$annihilations, as functions of $Q^{2}$ and $x_{p}$. The analysis is restricted to the current region of the Breit frame by boosting the tracks to this frame and requiring $P_{Z}^{\text {Breit }}<0$, where $P_{Z}^{\text {Breit }}$ is the longitudinal momentum of the track in the Breit frame.

The next-to-leading order (NLO) QCD predictions, based on different FFs, and leading-logarithm parton-shower MC calculations, interfaced with the Lund string fragmentation model, are compared to the measurements. Two sets of calculations based on different parameterisations of the FFs are used. The first set was obtained from fits to $e^{+} e^{-}$data and based on the program CYCLOPS [21], called "AKK+CYCLOPS" $[22,23]$. The second set was obtained from a global fit to $e^{+} e^{-}, p p$ and $e p$ data, called "DSS" [16].

Figure 7 shows the $M\left(\pi^{+} \pi^{-}\right)$and $M(p \pi)$ distributions. The data sample contains 806,505 $(165,875) K_{S}^{0}(\Lambda)$ candidates. In the current region of the Breit frame, there are $238,153 K_{S}^{0}$ and $40,728 \Lambda$ candidates.
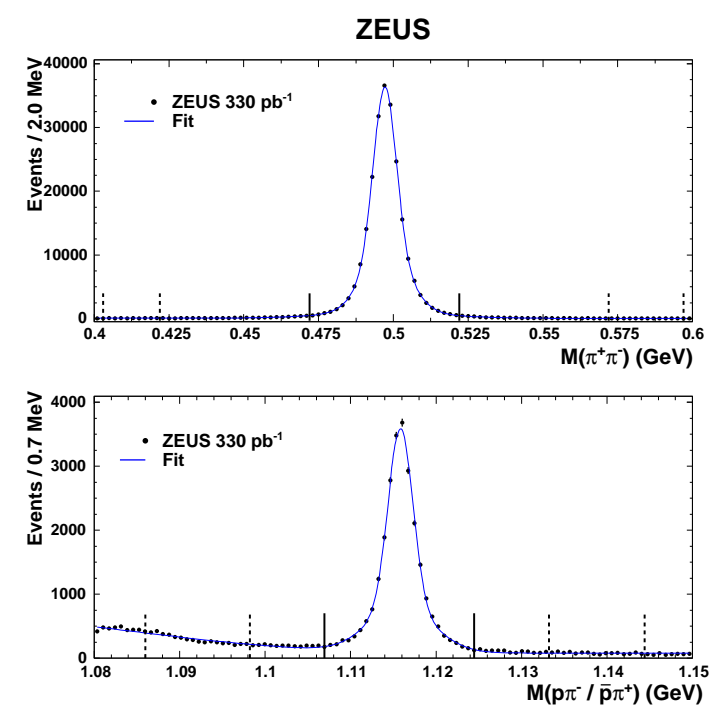

Figure 7: (a) The $\pi^{+} \pi^{-}$invariant-mass distribution for $K_{S}^{0}$ candidates. (b) The $p \pi^{-} / \bar{p} \pi^{+}$invariantmass distribution for $\Lambda / \bar{\Lambda}$ candidates. The solid line represents an indicative fit by two Gaussians and a (a) linear and (b) quadratic background function.

Scaled momentum distributions, $(1 / N)\left(n(H) / \Delta x_{p}\right)$, with $n(H)$ the number of $H\left(K_{S}^{0}\right.$ or $\left.\Lambda\right)$, $N$ the number of DIS events in a given $Q^{2}$ bin and $\Delta x_{p}$ the width of the $x_{p}$ bin, were measured in the current region of the Breit frame. The distributions are presented as functions of $Q^{2}$ and $x_{p}$ in the kinematic region of $10<Q^{2}<40000 \mathrm{GeV}^{2}$ and $0.001<x<0.75$. The left side of Figure 8 shows the scaled momentum distributions for $K_{S}^{0}$ as functions of $Q^{2}$ in different regions of $x_{p}$. The data show clear scaling violation as expected on the basis of the QCD description of the parton evolution with increasing $Q$ : the phase space for soft gluon radiation increases, leading to a rise of the number of soft particles with small $x_{p}$. The predictions from the CDM and MEPS models are compared to the measurements. They describe the shapes of the distributions fairly well while overestimating the overall production of $K_{S}^{0}$ by 10 to $20 \%$.

The NLO QCD calculations are also compared to the measurements for $x_{p}>0.1$. The AKK+CYCLOPS calculations predict a much too high $K_{S}^{0}$ rate but for $x_{p}>0.6$. These discrepancies might come from the fact that the FFs used in these predictions have a poorly constrained gluon contribution, which 


\section{ZEUS}
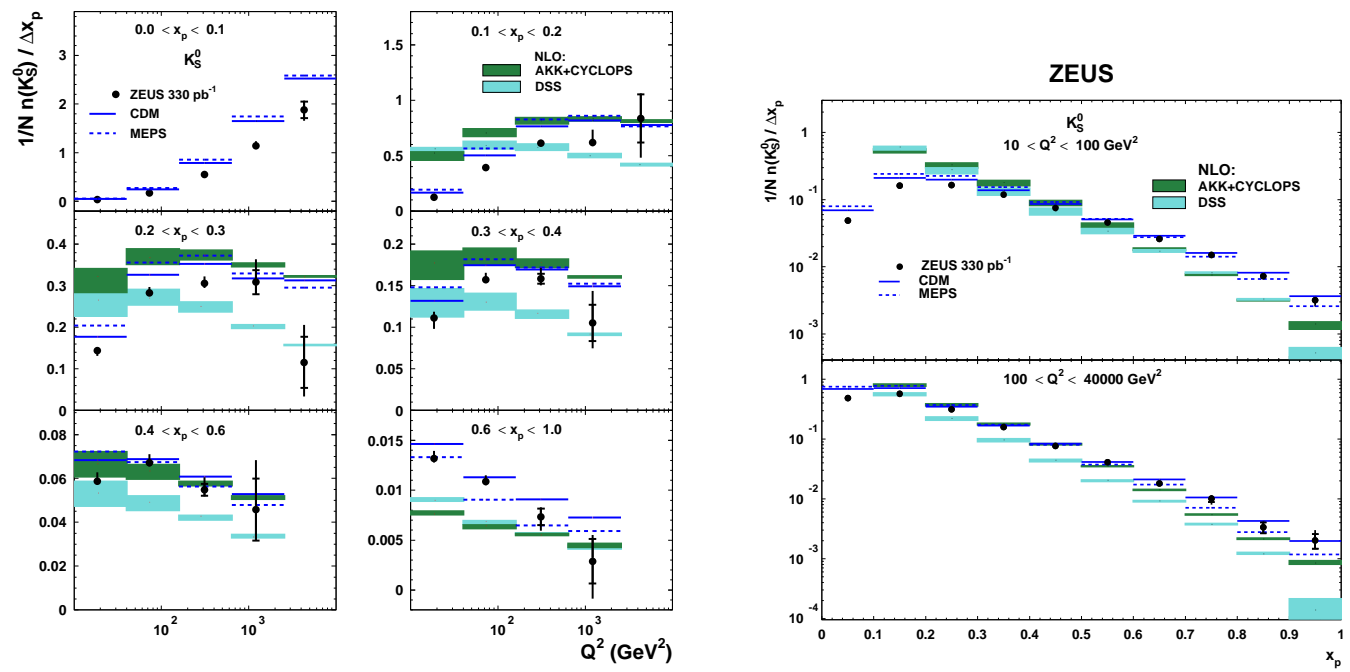

Figure 8: (Left) The measured scaled momentum distributions $(1 / N)\left(n\left(K_{S}^{0}\right) / \Delta x_{p}\right)$ as functions of $Q^{2}$ in different regions of $x_{p}$. For comparison, the NLO predictions of AKK+CYCLOPS and DSS are also presented. The bands represent the theoretical uncertainty. The predictions from CDM and MEPS MC simulations are also shown. (Right) The measured scaled momentum distributions $(1 / N)\left(n\left(K_{S}^{0}\right) / \Delta x_{p}\right)$ as functions of $x_{p}$ in different regions of $Q^{2}$ (dots).

is dominant at low $x_{p}$. The DSS calculations give a good description of the measurements for $x_{p}>0.3$ and $10<Q^{2}<40000 \mathrm{GeV}^{2}$. The prediction for this region of phase space is mainly constrained by $p p$ data, which sufficiently constrain the FFs at high $x_{p}$. At lower $x_{p}$, the DSS calculations fail to describe the data. This can be explained by the fact that the DSS fit in this region of phase space is mostly unconstrained by the available data. Thus, the measurements presented in this paper will help to improve significantly such global fits in this region of phase space. The right side of Figure 8 shows the scaled momentum distributions for $K_{S}^{0}$ as functions of $x_{p}$ in two regions of $Q^{2}$. The predictions of CDM and MEPS give a good description of the data. In both regions of $Q^{2}$, both NLO calculations predict too-steep spectra. At low $Q^{2}$, this effect is especially pronounced.

Figure 9 shows the scaled momentum distributions for $\Lambda$. Scaling violation is clearly observed. The predictions of CDM and MEPS give a reasonable description of the measurements, but overestimate the overall $\Lambda$ rate by $\approx 20 \%$. The AKK+CYCLOPS NLO calculations fail to describe the measurements. The predicted spectra in $x_{p}$ are, as in the case of $K_{S}^{0}$, significantly too steep.

Figure 10 shows the scaled momentum distributions for $K_{S}^{0}$ and $\Lambda$ together with those from the inclusive charged particles analysis [24] in the kinematic region of $0.1<x_{p}<0.4$ as functions of $Q^{2}$. For $Q^{2}>100 \mathrm{GeV}^{2}$ all distributions show a plateau. At lower $Q^{2}$ sizeable mass effects are clearly visible. For $0.1<x_{p}<0.2$, the value of $(1 / N)\left(n(H) / \Delta x_{p}\right)$ drops to $10(20) \%$ of its maximum value for $\Lambda\left(K_{S}^{0}\right)$, while for inclusive charged particles, the $(1 / N)\left(n(H) / \Delta x_{p}\right)$ value is still $40 \%$ of the plateau value at the lowest $Q^{2}$ accessible. 
ZEUS
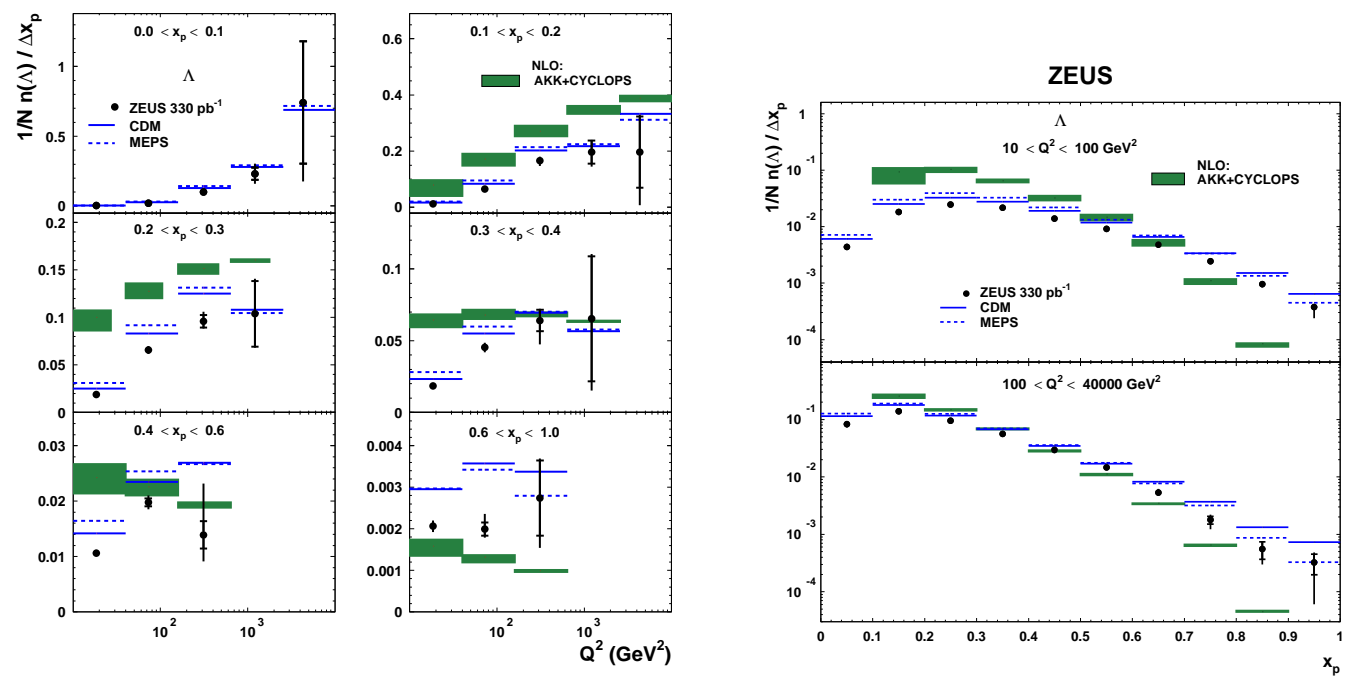

Figure 9: (Left) The measured scaled momentum distributions $(1 / N)\left(n(\Lambda) / \Delta x_{p}\right)$ as functions of $Q^{2}$ in different regions of $x_{p}$. (Right) The measured scaled momentum distributions $(1 / N)\left(n(\Lambda) / \Delta x_{p}\right)$ as functions of $x_{p}$ in different regions of $Q^{2}$. Other details as in the caption to Figure 9.

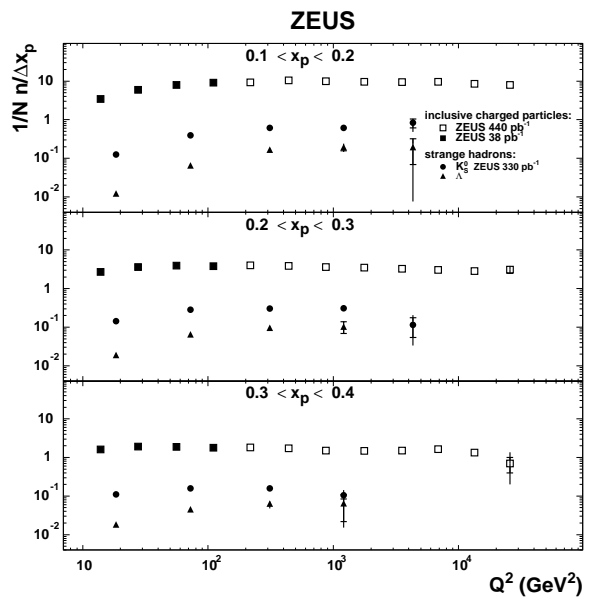

Figure 10: The measured scaled momentum distributions $(1 / N)\left(n(H) / \Delta x_{p}\right)$ for $H=K_{S}^{0}, \Lambda$ and light charged particles as functions of $Q^{2}$ in different regions of $x_{p}$. Other details as in the caption to Figure 9.

\subsection{Summary}

New measurements of $\mathrm{H} 1$ and ZEUS Collaborations on strange $K_{s}^{0}$ and $\Lambda$ hadron production in deep inelastic ep scattering at HERA are presented. The measurements are compared to MC model predictions based on the colour-dipole model (CDM) and DGLAP matrix element calculations supplemented with parton showers (MEPS). Within the uncertainties the models provide a reasonable description of the data. The sensitivity of the $K_{s}^{0}$ and $\Lambda$ cross sections to the strangeness suppression factor $\lambda_{s}$ is demonstrated. The $\Lambda-\bar{\Lambda}$ asymmetry is found to be consistent with zero.

Scaled momentum distributions for $K_{S}^{0}$ and $\Lambda$ hadrons were measured for the first time in $e p$ DIS. Scaling violations were clearly observed for both hadrons. The NLO QCD calculations based on different parameterisations of the FFs are compared to the measurements. The predictions based 
on FFs extracted from $e^{+} e^{-}$data alone fail to describe the measurements. The predictions based on a global analysis which include $e^{+} e^{-}, p p$ and $e p$ data give an improved description of the measurements. However, they predict a too high production rate of $K_{S}^{0}$ and $\Lambda$ hadrons at low $x_{p}$ and $Q^{2}$. The measurements presented here have the potential to constrain significantly the FFs for the strange hadrons $K_{S}^{0}$ and $\Lambda$.

\section{References}

[1] B. Andersson et al. Phys. Rept. 97 (1983) 31;

T. Sjöstrand, Comput. Phys. Commun. 39 (1986) 347.

[2] G. Altarelli et al., Nucl. Phys. B 160, 1979 (301).

[3] W. Furmanski and R. Petronzio, Z. Phys. C 11, 1982 (293).

[4] P. Nason and B.R. Webber, Nucl. Phys. B 421, 1994 (473).

[5] J.C. Collins and D.E. Soper, Nucl. Phys. B 193, 1981 (381), Nucl. Phys. B 213, 1983 (545).

[6] J.C. Collins and D.E. Soper, Nucl. Phys. B 194, 1982 (445).

[7] H1 Collaboration, " $K_{S}^{0}$ production at low $Q^{2}$ in Deep-Inelastic ep Scattering at HERA", H1prelim-13-033.

[8] H1 Collaboration, "Lambda baryons at high $Q^{2}$ at HERA", H1prelim-13-031.

[9] ZEUS Collaboration, H. Abramowicz et al., "Scaled Momentum Distributions for $K_{s}^{0}$ and $\Lambda / \bar{\Lambda}$ in DIS at HERA", JHEP 03 (2012) 020.

[10] K. Nakamura et al. (Particle Data Group), J. Phys. G 37 (2010) 075021.

[11] H. Jung, Comput. Phys. Commun. 86 (1995) 147.

[12] K. Charchula, G. A. Schuler, and H. Spiesberger, Comput. Phys. Commun. 81 (1994) 381.

[13] B. Andersson et al. Z. Phys. C 43, 1989 (625);

L. Lönnblad, Z. Phys. C 65, 1995 (285).

[14] L. Lönnblad, Comput. Phys. Commun. 71 (1992) 15.

[15] T. Sjöstrand, Comput. Phys. Commun. 82 (1994) 74.

[16] D. de Florian, R. Sassot and M. Stratmann, Phys. Rev. D 75, 2007 (114010).

[17] S. Albino, B.A. Kniehl and G. Kramer, Nucl. Phys. B 734, 2006 (50).

[18] F. Arleo, Eur. Phys. J. C 61, 2009 (603).

[19] R.P. Feynman, Photon-Hadron Interactions. Benjamin, New York, (1972)

[20] K.H. Streng, T.F. Walsh and P.M. Zerwas, Z. Phys. C 2, 1979 (237).

[21] D. Graudenz, Phys. Lett. B 406, 1997 (178).

[22] S. Albino et al., Phys. Rev. D 75, 2007 (034018).

[23] S. Albino, B.A. Kniehl and G. Kramer, Nucl. Phys. B 803, 2008 (42).

[24] ZEUS Collaboration, H. Abramowicz et al., JHEP 1006 (2010) 009. 